\title{
Structural, Electronic, and Magnetic Properties of $\mathrm{Mn}_{4} \mathrm{~N}$ Perovskite: Density Functional Theory Calculations and Monte Carlo Study
}

\author{
A. Azouaoui ${ }^{1}$ (D) M. El Haoua ${ }^{1}$ - S. Salmi ${ }^{1}$ - A. El Grini ${ }^{1} \cdot$ N. Benzakour ${ }^{1} \cdot$ A. Hourmatallah $^{2} \cdot$ K. Bouslykhane $^{1}$
}

Received: 21 August 2019 / Accepted: 23 October 2019 / Published online: 17 December 2019

(C) The Author(s) 2019

\begin{abstract}
In this paper, we have studied the structural, electronic, and magnetic properties of the cubic perovskite system $M n_{4} N$ using the first principles calculations based on density functional theory (DFT) with the generalized gradient approximation (GGA). The obtained data from DFT calculations are used as input data in Monte Carlo simulation with a mixed spin-5/2 and 1 Ising model to calculate the magnetic properties of this compound, such as the total, partial thermal magnetization, and the critical temperatures $\left(T_{C}\right)$. The obtained results show that $M n_{4} N$ has a ferrimagnetic structure with two different sites of $\mathrm{Mn}$ in the lattice and presents a metallic behavior. The obtained $T_{C}$ is in good agreement with experimental results.
\end{abstract}

Keywords Cubic perovskite $M n_{4} N$ - DFT $\cdot$ Monte Carlo simulation · Critical temperature

\section{Introduction}

Transition metal nitride (TMNs) materials have recently attracted much attention due to their excellent structural, electronic, and magnetic properties $[1,2]$ that can be applied to many fields, including magnetic recording [3, 4], spin-injection contacts [5, 6], and microwave-absorbing materials [7]. Perpendicular magnetic anisotropy (PMA) is one of the most properties found in $\mathrm{Mn} 4 \mathrm{~N}$ thin film of TMNs [8]; this property makes it suitable for applications in spintronics device such as nonvolatile memory [5, 9-11].

The mechanical stability of Mn4N bulk was investigated in [12-16]; among the four different crystal stable phases, namely, $\eta, \zeta, \theta$, and $\varepsilon, \varepsilon-M n_{4} N$ is the most stable structure.

The $M n_{4} N$ bulk is a ferrimagnetic metal with a high Curie temperature of $T_{N}=738 \mathrm{~K}$ and low magnetic moment [17]. The structure is cubic perovskite-type, crystallized with the face-centered cubic structure (space group $P m \overline{3} m$, No. 221). The unit cell contains five atoms,

\footnotetext{
A. Azouaoui

azouaoui01@yahoo.fr
}

1 Laboratoire de Physique du Solide, Université Sidi Mohammed Ben Abdellah, Faculté des sciences Dhar Mahraz, BP 1796, Fes, Morocco

2 Laboratoire LIPI, Equipe de Physique du Solide, Université Sidi Mohammed Ben Abdellah Ecole Normale Supérieure, Bensouda, BP 5206, Fes, Morocco one nitrogen atom $(\mathrm{N})$ at the body center positions $(0.5,0.5$, $0.5)$, and four manganese atoms (Mn) distributed with two inequivalent magnetic sites. One at the corners labelled $M n_{c}$ (cube corner position $(0,0,0))$ and the other at the facecentered labelled $M n_{f}$ (cube face $(0.5,0.5,0),(0.5,0,0.5)$, and $(0,0.5,0.5)$ positions) (Fig. 1). The exchange coupling of both magnetic sites is antiferromagnetic [18].

In this paper, we study the structural, electronic, and magnetic properties of $M n_{4} N$. These properties have been investigated using density functional theory (DFT) implemented in Quantum ESPRESSO package. The obtained data from DFT calculations are used in Monte Carlo simulation with a mixed spin-5/2 and 1 Ising model to study the thermal magnetization and magnetic susceptibility and calculate the critical temperature $\left(T_{C}\right)$.

\section{Computational Details}

All the calculations are performed in the framework of the density functional theory (DFT), as implemented in the QUANTUM ESPRESSO package [19]. The exchange and correlation energies were calculated by the generalized gradient approximation (GGA) using the Perdew-BurkeErnzerhof (PBE) gradient corrected functional [20]. The Vanderbilt ultrasoft pseudopotentials [21] were used to treat the valence electrons for $\mathrm{Mn}\left(3 d^{5} 4 s^{2}\right)$ and $\mathrm{N}\left(2 s^{2} 2 p^{3}\right)$. Brillouin zone integration has been done using a MethfesselPaxton [22] smearing of $0.01 \mathrm{Ry}$ and a special k-points 
Fig. 1 The perovskite structure of $M n_{4} N$ compound. The large balls depict manganese atoms, and the small blue ball represents the nitride atom

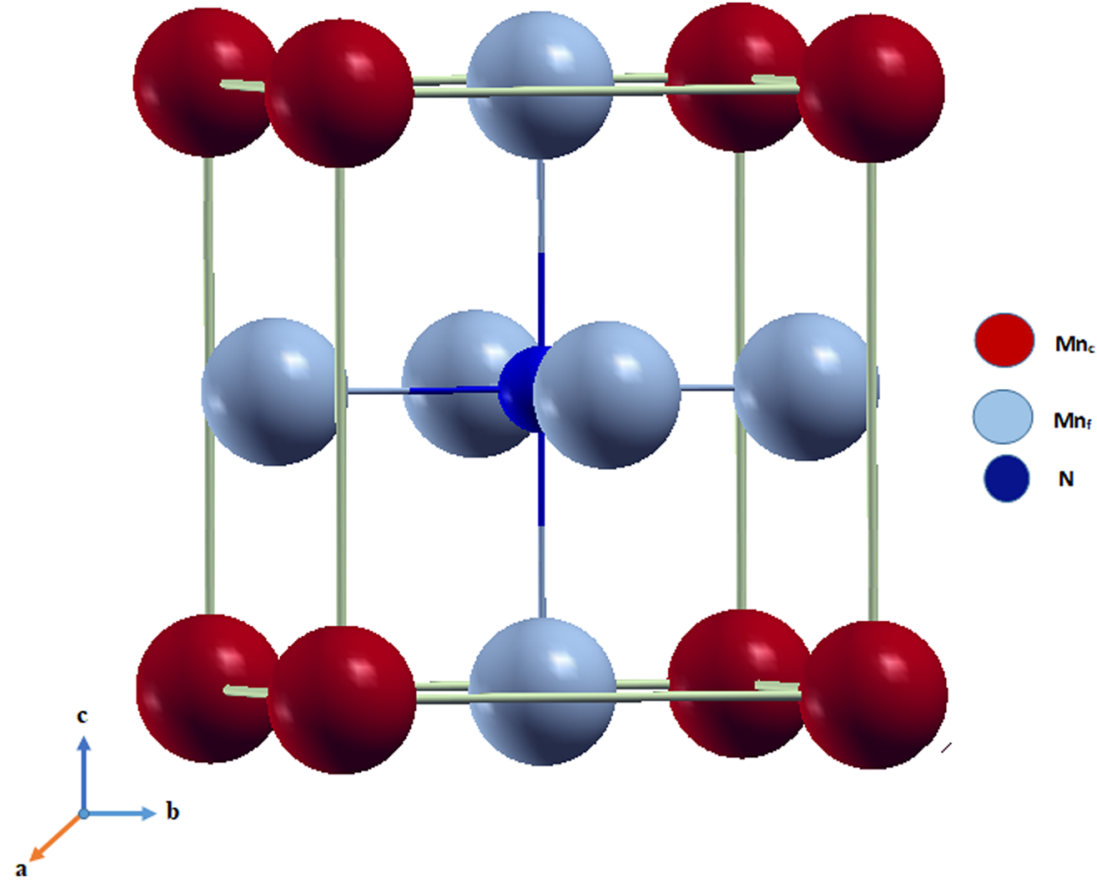

grid of $6 \times 6 \times 6$ [23]. Khon-Sham states are expanded in plane waves with a kinetic energy cutoff of $520 \mathrm{eV}$, and the charge-density cutoff is kept at 8 times that of the kinetic energy cutoff.

\section{Theory and Model}

The Hamiltonian of the system with a ferrimagnetic spin$5 / 2$ and 1 configuration including the nearest neighbors interactions and an external magnetic field is given by:

$H=-J_{\sigma S} \sum_{<i j>} \sigma_{i} S_{j}-h_{e x}\left(\sum_{i} \sigma_{i}+\sum_{j} S_{j}\right)$

where $<i j>$ stands for the first nearest neighbor spins ( $\mathrm{i}$ and $\mathrm{j}$ ) and $h_{e x}$ is the external magnetic field, and $J_{\sigma S}$ is the exchange interactions between the first nearest neighbor magnetic atoms with the spins $\sigma$ and $\mathrm{S}$. The possible projections spin moment of atom $M n_{f}$ are $\sigma=-1,0,1$ and of $M n_{c}$ are $S= \pm \frac{5}{2}, \pm \frac{3}{2}, \pm \frac{1}{2}$.

\section{Monte Carlo Study}

The $M n_{4} N$ is formed by two types of spins $\sigma$ and S. The total number of spins $N=N_{\sigma}+N_{S}$ with $N_{\sigma}=375$ and $N_{S}=125$. The Monte Carlo simulation reported in [24] is applied to simulate the Hamiltonian
H. We have used the cyclic boundary conditions on the lattice which were imposed and were generated by sequentially traversing the lattice and making single-spin flip attempts. The Monte Carlo update was performed by choosing random spins and then flipped to opposite state with Boltzmann-based probability. In general, this can be done using the conventional Metropolis algorithm [25]. The flips are accepted or rejected according to a heat-bath algorithm under the Metropolis approximation. Our data were generated with $10^{5}$ Monte Carlo steps per spin, discarding the first $10^{4}$ Monte Carlo simulations. Our program calculates the following parameters:

The magnetizations of each atom of manganese $M n_{c}$ and $M n_{f}$ in $M n_{4} N$ structure are given by:

$$
\begin{aligned}
& M_{\sigma}=\frac{1}{N_{\sigma}}\left\langle\sum_{i} \sigma_{i}\right\rangle \\
& M_{S}=\frac{1}{N_{S}}\left\langle\sum_{i} S_{i}\right\rangle
\end{aligned}
$$

The total magnetization is given by:

$$
M_{t o t}=\frac{N_{\sigma} M_{\sigma}+N_{S} M_{S}}{N_{\sigma}+N_{S}}
$$

The internal energy E per site:

$$
E=\frac{1}{N_{S}+N_{\sigma}}\langle H\rangle
$$


The magnetic susceptibilities:

$$
\begin{aligned}
\chi_{\sigma} & =\beta\left(<M_{\sigma}^{2}>-<M_{\sigma}>^{2}\right) \\
\chi_{S} & =\beta\left(<M_{S}^{2}>-<M_{S}>^{2}\right) \\
\chi_{t o t} & =\frac{N_{\sigma} \chi_{\sigma}+N_{S} \chi_{S}}{N_{\sigma}+N_{S}}
\end{aligned}
$$

where $\beta=\frac{1}{k_{B} T}$ and $\mathrm{T}$ denotes the absolute temperature.

\section{Results and Discussion}

We have optimized the lattice parameter for two magnetic configurations: ferromagnetic ( $\uparrow \uparrow \uparrow)$ all spins are up and parallels, antiferromagnetic $(\uparrow \downarrow \downarrow \downarrow)$, one atom at the corner $M n_{c}$ with the spin up, and three atoms at the face-centered $M n_{f}$ with the spin down. The total energies variation as a function of lattice parameter obtained by using the local spin density approximation (LSDA) and (GGA) and fitting with Murnaghan's equation of state [26] are presented in Fig. 2.

The formation energy $E_{f}$ per formula unit of manganese nitride can be expressed by [27]:

$E_{f}=E\left(M n_{4} N\right)-\left(4 E(M n)+E\left(N_{2}\right) / 2\right)$

where $E\left(M n_{4} N\right)$ is the total energy of $M n_{4} N$ bulk, $E(M n)$ the total energy of the Mn pure metal, and $E\left(N_{2}\right)$ is the total energy of the $N_{2}$ dimer at equilibrium lattice constant. The values of the optimized lattice parameter, total energy, bulk modulus, and formation energy are listed in Table 1.

Table 1 shows that the FIM ( $\downarrow \downarrow \downarrow \downarrow)$ configuration is the most stable state in energy than FM configuration. The lattice constant obtained for FIM ( $\downarrow \downarrow \downarrow \downarrow)$ configuration is $3.7475 \AA$. This value is in good agreement with other published theoretical works [28, 30], and less than the value (3.86§) reported experimentally in ref. [29]. The results listed in Table 1 show that $M n_{4} N$ is a hard material with 402.4 GPa and the hardness decreases from 490.0 GPa for ferromagnetic configuration to $402.4 \mathrm{GPa}$ for ferrimagnetic configuration [28].

The calculated total and local magnetic moments in Bohr magnetons $\left(\mu_{B}\right)$ per formula unit of $M n_{4} N$ for both FM and FIM configurations are listed in Table 2. Our obtained values in ferrimagnetic configuration $(\uparrow \downarrow \downarrow \downarrow)$ are in good agreement with the experiment ones reported in ref. [31], with the moment of $\mu_{M n_{c}}=3.50 \mu_{B}$ and the moment of $\mu_{M n_{f}}=-0.8686 \mu_{B}$. The results listed in Table 2 show clearly that the $M n_{4} N$ is ferrimagnetic material with low magnetic moment [17].

We have presented in Fig. 3 the variations of the magnetic moment of $M n_{f}$ (Fig 3a) and $M n_{c}$ (Fig 3b) versus lattice parameter. The magnetic moments increase when the lattice
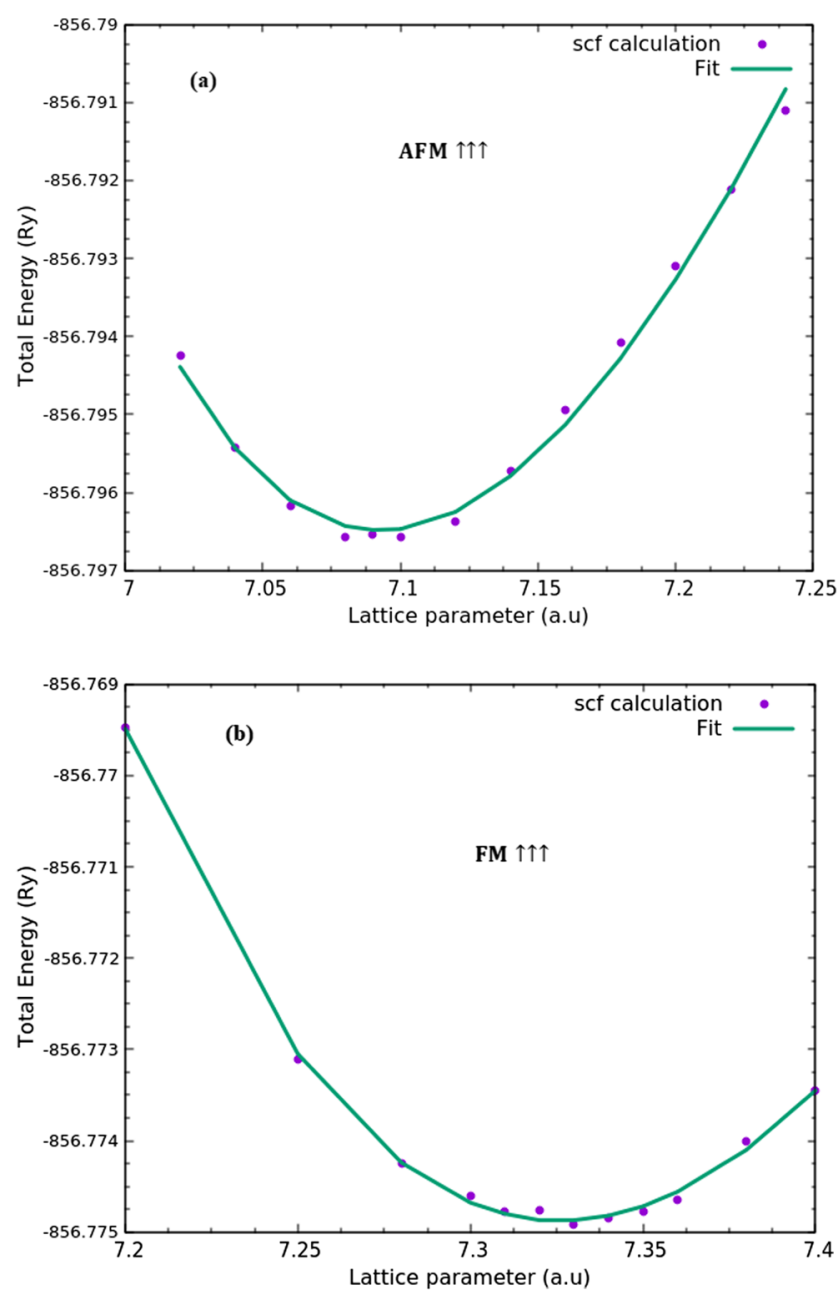

Fig. 2 Total energy versus lattice parameter: a with AFM configuration and $\mathbf{b}$ with FM configuration

parameter increases, and in the vicinity of the optimal lattice parameter, both magnetic moments have large deviations.

The total moment per formula unit for the $M n_{4} N$ system with cubic structure can be expressed by the following expression:

$\mu_{t o t}=\mu_{M n_{c}}+3 \mu_{M n_{f}}$.

The expected value of $\mu_{t o t}$ is $0.5 \mu_{B}$. Our obtained value in this work is $0.76 \mu_{B}$. This value is greater than $0.5 \mu_{B}$ and less than that obtained experimentally $\left(\mu_{\exp }=0.9 \mu_{B}\right)$ [31].

The total and partial density of state and band structure for $M n_{4} N$, deduced from the band structure calculations using GGA functional for the FIM configuration, at equilibrium lattice constant and along the high symmetry lines of the Brillouin zone for band structure are depicted in Figs. 4 and 5. Here, the zero energy corresponds to the Fermi level. The DOS characterized by an asymmetry of spin up and spin down contributions and dominated by the 
Table 1 Configuration, lattice constants, total energy, bulk modulus, and the formation energy per formula unit

\begin{tabular}{|c|c|c|c|c|}
\hline Configuration & a $(\AA)$ & Total energy (Ry) & $\mathrm{B}(\mathrm{GPa})$ & Formation energy $(\mathrm{eV})$ \\
\hline $\mathrm{FM}(\uparrow \uparrow \uparrow \uparrow)$ & 3.876 & -856.77483 & 490.0 & -1.7 \\
\hline FIM $(\uparrow \downarrow \downarrow \downarrow)$ & 3.748 & -856.79649 & 402.4 & -1.78 \\
\hline Other works [28] & 3.744 & & 219.8 & -1.47 \\
\hline Experiment [29] & 3.86 & & & \\
\hline
\end{tabular}

Mn atom originate essentially from contributions of 3D band which shows that ferrimagnetic behavior.

The projected density of state (PDOS) for $M n_{c}$ and $M n_{f}$ is depicted in Fig. 6 a and b, respectively. These PDOS provide more detailed picture on the $M n_{4} N$ compound and show that the largest contribution close to the Fermi level comes from the $d_{x y}$ and $d_{z^{2}}$ orbital and the $N-p$ orbitals have only slight contributions.

The band structure and DOS of $M n_{4} N$ show that the Fermi level is populated mainly by the $M n-d$ orbitals and demonstrates that $M n_{4} \mathrm{~N}$ has a metallic behavior.

In order to investigate the magnetic properties of $\mathrm{Mn}_{4} \mathrm{~N}$ system by mean field theory and Monte Carlo simulations. We have studied the magnetic interactions between ions of Mn by first principles calculations using the generalized gradient approximation (GGA). The energy difference between the ferrimagnetic and ferromagnetic orders can be expressed by $[32,33]$ :

$$
\Delta E=E_{F I M}-E_{F M}
$$

where $E_{F I M}$ and $E_{F M}$ are the total energy of ferrimagnetic and ferromagnetic states, respectively.

Based on the mean field theory (MFT) and the method used by Holland and Brown in Ref. [34]. The Curie temperature of $M n_{4} N$ system can be given approximately by:

$$
k_{B} T_{C}^{M F T}=\frac{1}{3} \sum_{i \neq 0} J_{0 i}
$$

Table 2 Total and local moment, in Bohr magnetons $\left(\mu_{B}\right)$, per formula

\begin{tabular}{|c|c|c|c|}
\hline Configuration & $\mu_{t o t}$ & $\mu_{M n_{c}}$ & $\mu_{M n_{f}}$ \\
\hline $\mathrm{FM}(\uparrow \uparrow \uparrow \uparrow)$ & 11.12 & 3.1437 & 2.2453 \\
\hline FiM $(\uparrow \downarrow \downarrow \downarrow)$ & 0.76 & 3.5076 & -0.8686 \\
\hline
\end{tabular}
unit
Where the sum extends over all the first neighbor atoms and $k_{B}$ is the Boltzmanns constant $\left(k_{B}=\right.$ $\left.8,617330.10^{-5} \mathrm{eV} \mathrm{K}^{-1}\right)$.

$k_{B} T_{C}^{M F T}=\frac{1}{3} z J_{\sigma S} S \sigma$

$\mathrm{z}$ is the number of first nearest neighbors around $\mathrm{Mn}$ atom $(\mathrm{z}=12)$.
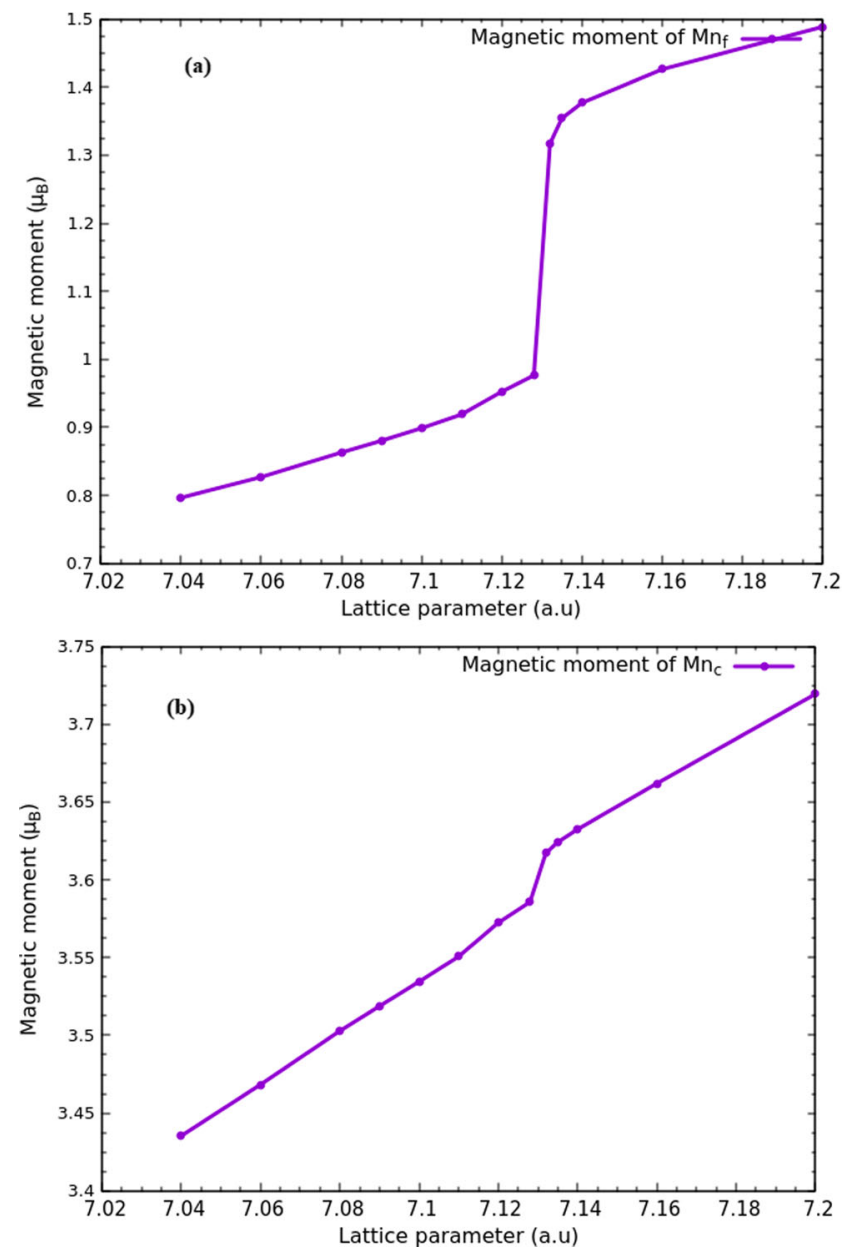

Fig. 3 Magnetic moment versus lattice parameter: $\mathbf{a}$ of $M n_{f}$ and $\mathbf{b}$ of $M n_{c}$ 


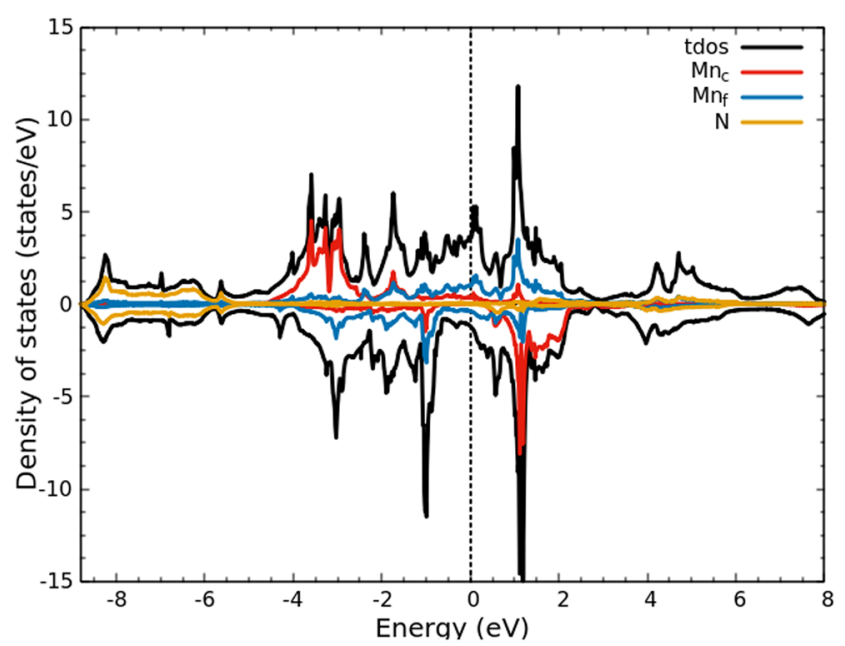

Fig. 4 Total and partial dos of $\mathrm{Mn}_{4} \mathrm{~N}$

Therefore, $T_{C}$ can be given as a function of $\Delta E$ by:

$T_{C}=\frac{\Delta E}{6 K_{B}}=\frac{\left(E_{F i M}-E_{F M}\right)}{6 k_{B}}$

where the energy difference is $\Delta E=0.53 \mathrm{eV}$

The exchange interaction can be expressed as a function of $\Delta E$ by the following expression:

$\frac{J_{\sigma S}}{k_{B}}=\frac{\Delta E}{2 z \sigma S}$

The obtained value of the exchange parameter with DFT calculation is used as input for Monte Carlo simulation to calculate the critical temperature.

Figures 7 and 8 show the total and partial magnetization of and magnetic susceptibility of each atom of manganese $M n_{c}$ and $M n_{f}$ with spins $S=5 / 2$ and $\sigma=1$, with zero external magnetic fields $\left(h_{e x}=0\right)$. The transition from ferrimagnetic phase to the paramagnetic one occurs at the Curie temperature $T_{C}=705 \mathrm{~K}$, is deduced from

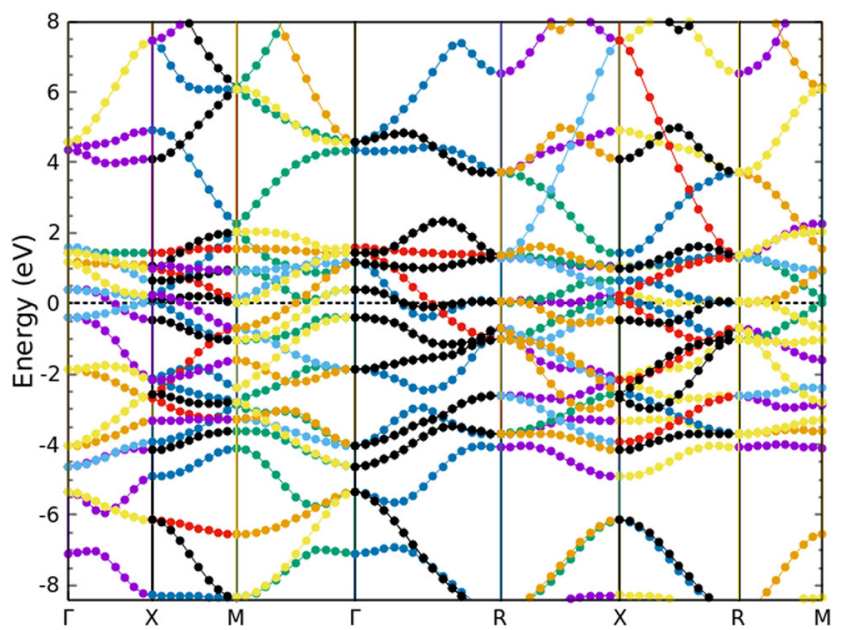

Fig. 5 Bands structure of $M n_{4} N$
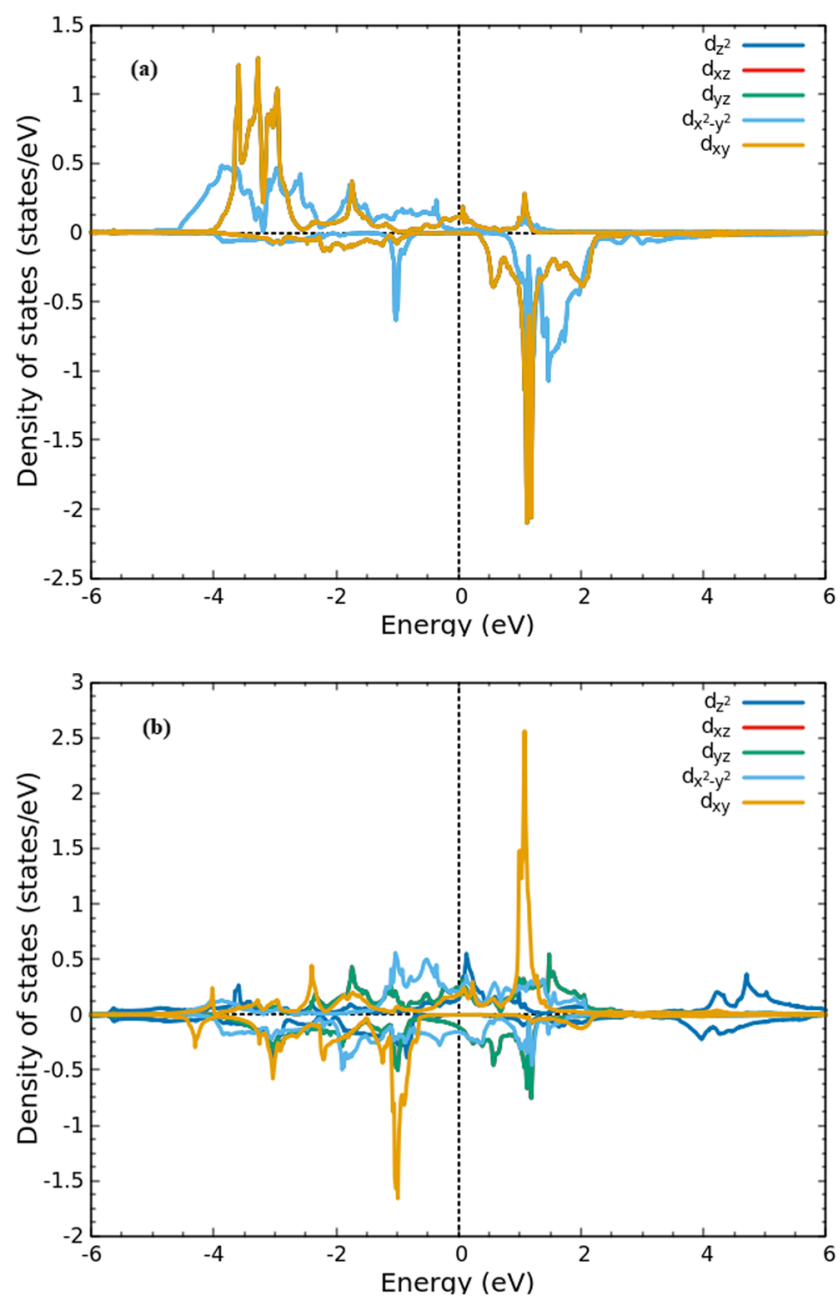

Fig. 6 projected dos of $M n-d$ orbital: $\mathbf{a}$ at the corner and $\mathbf{b}$ at the face

the maximum of magnetic susceptibility in Fig. 8. The obtained value of $T_{C}$ is in good agreement with that obtained by Monte Carlo simulation in ref. [30]. This value is

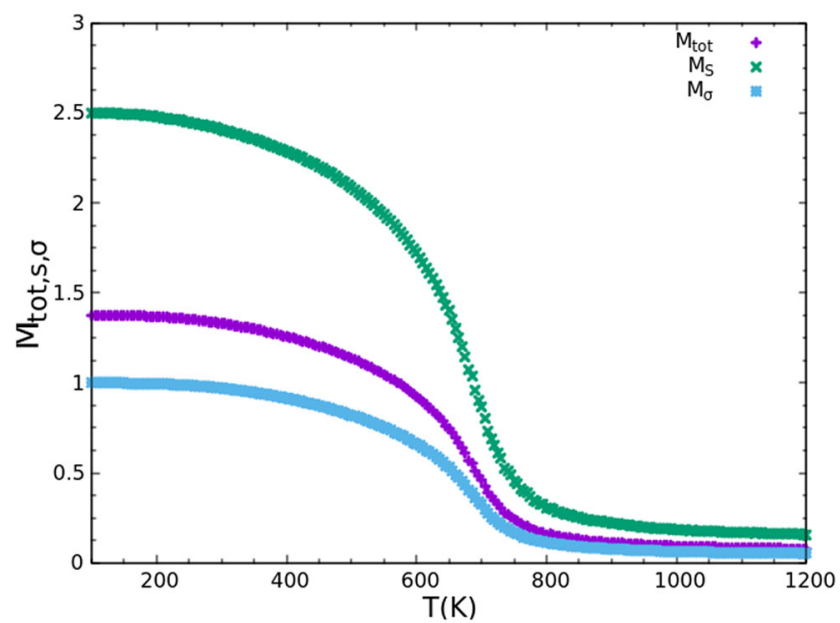

Fig. 7 The total and partial magnetization of $M n_{4} N$ for $h_{e x}=0$ 


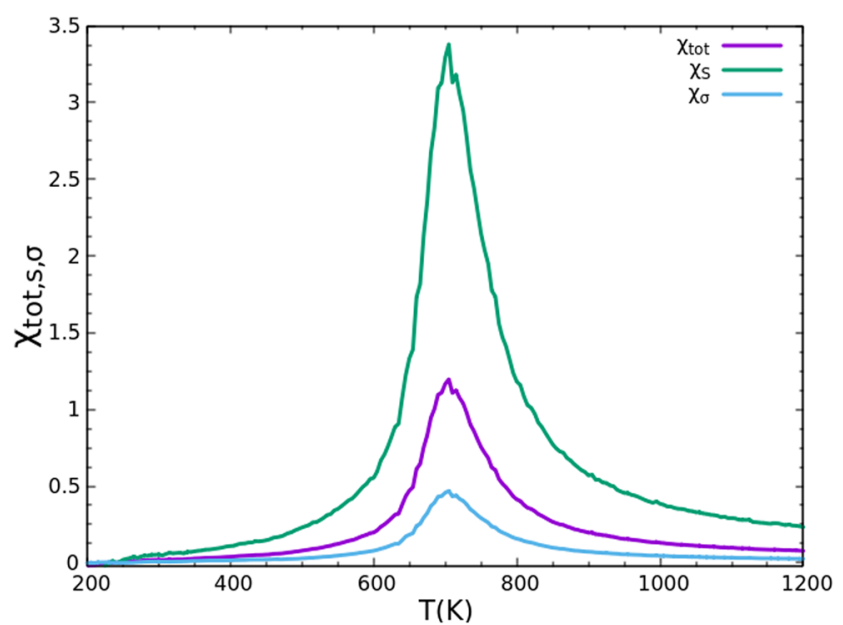

Fig. 8 The total and partial magnetic susceptibility of $M n_{4} N$ for $h_{e x}=0$

smaller than those obtained within MFT $\left(T_{C}^{M F T}=1025 K\right)$ and comparable with the experimental values obtained in previous works $[17,35]$.

\section{Conclusion}

Structural, electronic, and magnetic properties of $\mathrm{Mn}_{4} \mathrm{~N}$ with cubic perovskite structure have been investigated by first principles calculations of density functional theory. The results show that the $M n_{4} N$ has a metallic behavior with a ferrimagnetic arrangement of spins and a low magnetic moment. The density of state study reveals that the DOS around the Fermi level originates mainly from the 3D band contributions of $\mathrm{Mn}$. The critical temperature obtained by Monte Carlo simulation with mixed spin-5/2 and 1 Ising model is in good agreement with the experimental results.

Open Access This article is distributed under the terms of the Creative Commons Attribution 4.0 International License (http:// creativecommons.org/licenses/by/4.0/), which permits unrestricted use, distribution, and reproduction in any medium, provided you give appropriate credit to the original author(s) and the source, provide a link to the Creative Commons license, and indicate if changes were made.

\section{References}

1. Li, C., Yang, Y., Lv, L., Huang, H., Wang, Z., Yang, S.: J. Alloys Compd. 457(1-2), 57-60 (2008)

2. Fruchart, D., Givord, D., Convert, P., L'Heritier, P.H., Senateur, J.P.: J. Phys. F: Metal Phys. 9(12), 2431 (1979)

3. Jia, H., Wang, X., Zheng, W., Chen, Y., Feng, S.: Materials Science and Engineering: B 150(2), 121-124 (2008)
4. Linnik, A.I., Prudnikov, A.M., Shalaev, R.V., Linnik, T.A., Varyukhin, V.N., Kostyrya, S.A., Burkhovetskii, V.V.: Tech. Phys. Lett. 39(2), 143-146 (2013)

5. Shen, X., Chikamatsu, A., Shigematsu, K., Hirose, Y., Fukumura, T., Hasegawa, T.: Appl. Phys. Lett. 105(7), 072410 (2014)

6. Anzai, A., Gushi, T., Komori, T., Honda, S., Isogami, S., Suemasu, T.: J. Appl. Phys. 124(12), 123905 (2018)

7. Duan, Y., Xi, Q., Zhang, Y., Wen, M., Wang, T.: Appl. Phys. A 120(3), 1075-1081 (2015)

8. Yasutomi, Y., Ito, K., Sanai, T., Toko, K., Suemasu, T.: J. Appl. Phys. 115(17), 17A935 (2014)

9. Parker, F.T., Takano, K., Berkowitz, A.E.: Phys. Rev. B 61(2), R866 (2000)

10. Anzai, A., Takata, F., Gushi, T., Toko, K., Suemasu, T.: J. Cryst. Growth 489, 20-23 (2018)

11. Komori, T., Anzai, A., Gushi, T., Toko, K., Suemasu, T.: J. Cryst. Growth 507, 163-167 (2019)

12. Feng, W.J., Sun, N.K., Du, J., Zhang, Q., Liu, X.G., Deng, Y.F., Zhang, Z.D.: Solid State Commun. 148(5-6), 199-202 (2008)

13. Liu, Z.T.Y., Zhou, X., Khare, S.V., Gall, D.: Journal of Physics: Condensed Matter 26(2), 025404 (2013)

14. Liu, Z.T.Y., Zhou, X., Gall, D., Khare, S.V.: Comput. Mater. Sci. 84, 365-373 (2014)

15. Zhou, X., Gall, D., Khare, S.V.: J. Alloys Compd. 595, 80-86 (2014)

16. Guerrero-Sánchez, J., Takeuchi, N.: Appl. Surf. Sci. 407, 209-212 (2017)

17. Mekata, M.: J. Phys. Soc. Japan 17(5), 796-803 (1962)

18. Takei, W.J., Shirane, G., Frazer, B.C.: Phys. Rev. 119(1), 122 (1960)

19. Giannozzi, P., Baroni, S., Bonini, N., Calandra, M., Car, R., Cavazzoni, C., Ceresoli, D., Chiarotti, G.L., Cococcioni, M., Dabo, I., et al.: Journal of Physics: Condensed Matter 21(39), 395502 (2009)

20. Perdew, J.P., Burke, K., Ernzerhof, M.: Phys. Rev. Lett. 77(18), 3865 (1996)

21. Vanderbilt, D.: Phys. Rev. B 41(11), 7892 (1990)

22. Methfessel, M.P.A.T., Paxton, A.T.: Phys. Rev. B 40(6), 3616 (1989)

23. Monkhorst, H.J., Pack, J.D.: Phys. Rev. B 13(12), 5188 (1976)

24. Masrour, R., Jabar, A., Hlil, E.K.: J. Magn. Magn. Mater. 453, 220-225 (2018)

25. Metropolis, N., Rosenbluth, A.W., Rosenbluth, M.N., Teller, A.H., Teller, E.: The J. Chem. Phys. 21(6), 1087-1092 (1953)

26. Murnaghan, F.D.: Proceedings of the National Academy of Sciences of the United States of America 30(9), 244 (1944)

27. Fang, C.-M., Koster, R.S., Li, W.-F., van Huis, M.A.: RSC Adv. 4(16), 7885-7899 (2014)

28. Adhikari, V., Liu, Z.T.Y., Szymanski, N.J., Khatri, I., Gall, D., Sarin, P., Khare, S.V.: J. Phys. Chem. Solids 120, 197-206 (2018)

29. Foley, A., Corbett, J., Richard, A.L., Alam, K., Ingram, D.C., Smith, A.R.: J. Cryst. Growth 446, 60-67 (2016)

30. Meinert, M.: Journal of Physics: Condensed Matter 28(5), 056006 (2016)

31. Takei, W.J., Heikes, R.R., Shirane, G.: Phys. Rev. 125(6), 1893 (1962)

32. Musa, M., Saad, H.-E.: J. Phys. Chem. Solids 94, 1-11 (2016)

33. Zhao, H.J., Liu, X.Q., Chen, X.M.: AIP Adv. 2(2), 9 (2012)

34. Holland, W.E., Brown, H.A.: Physica Status Solidi (A) 10(1), 249-253 (1972)

35. Pop, I., Andrecut, M., Burda, I., Munteanu, R.: Materials Chem. Phys. 37(1), 52-54 (1994)

Publisher's Note Springer Nature remains neutral with regard to jurisdictional claims in published maps and institutional affiliations. 